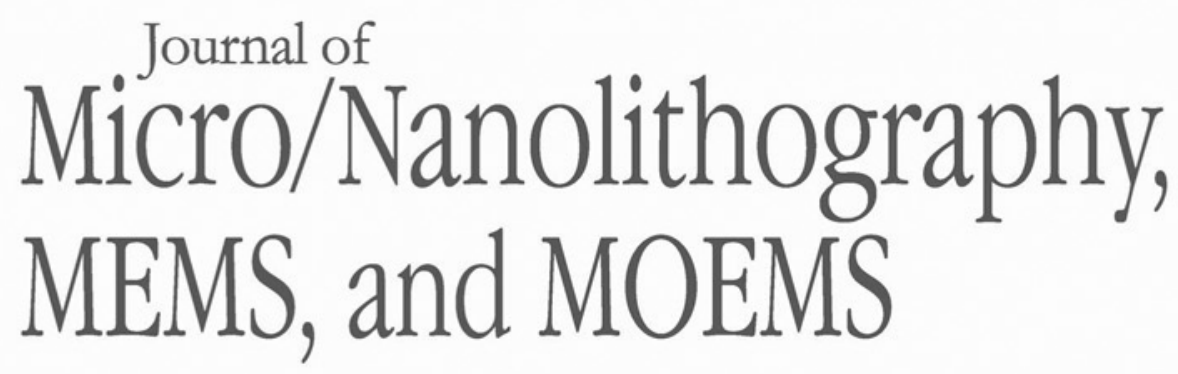

Nanolithography.SPIEDigitalLibrary.org

\title{
Current understanding of the electrostatic risk to reticles used in microelectronics and similar manufacturing processes
}

Gavin C. Rider 


\title{
Current understanding of the electrostatic risk to reticles used in microelectronics and similar manufacturing processes
}

\author{
Gavin C. Rider* \\ Microtome Inc., Colorado Springs, Colorado, United States
}

\begin{abstract}
This paper explains how an electric field and a reticle interact and describes the different kinds of damage that can be caused to a reticle through its exposure to electric field. It is shown why electrostatic reticle damage has changed from ESD damage (which causes yield to suddenly drop precipitously) into a gradual and cumulative form of degradation that is very difficult to diagnose. It is explained why some of the approaches that have been taken to reduce ESD damage in the semiconductor factory, such as equipotential bonding and the use of static dissipative plastics for making reticle pods, actually increase the risk of this cumulative type of electrostatic degradation in reticles. When assessing the risk to reticles and designing an effective protective strategy for reticle handling, it is shown why one must take into account the temporal characteristics of a reticle's interaction with electric field-including the effect that the reticle's immediate surroundings will have on that interaction-as well as considering the strength of any electric field in the reticle handling environment. Solutions are presented that would allow the electrostatic risk to reticles to be reduced significantly, without requiring major changes to operating procedures in semiconductor manufacturing facilities. @ 2018 Society of Photo-Optical Instrumentation Engineers (SPIE) [DOI: 10.1117/1.JMM.17.2.020901]
\end{abstract}

Keywords: Reticle ESD; progressive defects; reticle damage; electrostatic damage; EFM; CD degradation; chrome migration; reticle protection; yield loss.

Paper 18009V received Jan. 17, 2018; accepted for publication Mar. 27, 2018; published online Apr. 27, 2018.

\section{Introduction}

Measures to reduce the generation of static electricity in semiconductor production facilities are now widely adopted, in part to prevent electrostatic discharges (ESD) during material handling. While this has reduced the electrostatic risk to reticles, it has also changed the nature of the damage caused; from ESD that causes yield to drop precipitously into a more gradual and cumulative degradation process. The progressive miniaturization of pattern features has also changed the nature of the damage in the same way, which makes it look as if the electrostatic countermeasures that have been adopted are working correctly. That is an unfortunate misinterpretation of the situation, which this paper aims to correct.

Static electricity in the semiconductor production environment has been a problem for decades. In the 1990s, electrostatic damage to reticles became increasingly severe, causing significant disruption to production schedules and with financial losses from a single reticle damage event ranging from a few thousand up to hundreds of thousands of dollars. Those losses were significant enough to justify the investment of millions of dollars into the development and implementation of damage prevention technologies, but these ESD countermeasures were implemented before there was a complete understanding of all the physical mechanisms that operate when a reticle interacts with an electric field. Unfortunately, some of the protective measures that

*Address all correspondence to: Gavin C. Rider, E-mail: gavinrider@microtome .com have been adopted, while being appropriate for the protection of sensitive electronic devices against ESD during manufacture and handling, are not appropriate for the protection of reticles.

Reticles are uniquely sensitive to electric field and it is the management of electric field rather than electrical potential that must be optimized to ensure reticle safety.

One widely adopted practice involves connecting the reticle to ground potential through a static dissipative (i.e., resistive) contact, which is a standard procedure called equipotential bonding. Controlling the electrical potential of a reticle in this way seems entirely logical; it is done to prevent charge transfer and consequential ESD damage from taking place when moving the reticle from one place to another. However, controlling the reticle's electrical potential in this way does not prevent electric field from interacting with it. In fact, grounding a reticle concentrates any electric field that may be present in the reticle's handling environment directly through the reticle pattern. ${ }^{1,2}$ Electric field induces charge redistribution within the reticle and this is what causes damage, as explained in the following section. It has been shown by computer simulation and confirmed experimentally that grounding a reticle enhances field induction, ${ }^{3,4}$ thereby increasing the risk and the severity of any field-induced damage.

The importance of this becomes apparent when one realizes that electric fields are continually being generated around a reticle during its normal use. A reticle may be completely neutral, seemingly safely enclosed inside a closed reticle pod on a grounded storage shelf and not even being touched, but if an electric field is generated nearby, 
such as by an operator or a piece of electrical equipment, the reticle will amplify and concentrate the field and could be damaged by it. Furthermore, the reticle itself can easily become tribocharged during normal use. Bringing a charged reticle close to grounded equipment panels or handling tools also increases the field induction within the reticle, thereby increasing the risk of damage. ${ }^{2,5}$ Practices that are designed to reduce these risks are described in SEMI Standard E163. ${ }^{6}$

A second widely adopted practice that has some unforeseen negative consequences for reticles is the use of static dissipative plastic for making reticle pods. This development was undertaken after it was found that static charge accumulating on an insulating plastic single-reticle pod while an operator was carrying it could induce ESD in the reticle when the pod was placed onto a grounded load port and opened (the same is true of storage boxes and transit cases). Reducing the ability of the reticle container to accumulate static charge does indeed reduce the risk of ESD under those particular circumstances, but static dissipative plastic is not completely protective for the reticle. Static dissipative plastics have an undesirable "high pass" transmission characteristic for electric field; they block static and slowly varying electric fields but transmit rapidly changing fields. This has shifted the risk away from ESD toward a cumulative form of reticle damage that is more difficult to diagnose and which has more serious consequences in semiconductor production.

\section{How Electric Field Interacts with a Reticle}

When an electric field passes through a reticle, the electrons in the conductive features move virtually instantaneously in response to the field (an "electric field" is simply a description of the direction and magnitude of the force that a unit of charge will experience at each point in space- - the stronger the field, the greater the force). Each isolated conductive feature in the image area has an electrical potential induced on it that is determined by the strength of the electric field and its orientation relative to the features. The electric field becomes concentrated between some features and reduced between others, as shown by the computer simulation in Fig. 1.

Here, the gray features represent the conductive lines in a reticle pattern with the area at the right representing the solid border that normally surrounds the image area. A uniform electric field with a strength represented by the pale blue background passes from left to right. The simulation shows that, when the reticle pattern is introduced into this uniform field, the local field strength is strongly modified by the reticle's conductive features. The field strength is reduced in some places and becomes concentrated mainly at the ends of long lines, especially between features close to the edge of the pattern. The ambient field strength can be amplified by several orders of magnitude by the reticle's structure, as shown in the circled areas, which means that having even a very low level of electric field in the environment through which a reticle passes could be enough to cause damage within the reticle. ${ }^{7-10}$

As charge is displaced within the reticle under the influence of an applied electric field, it begins to generate an opposite internal electric field to the one that is being applied externally. The charge within the reticle moves until the opposing electric field thus created exactly balances and cancels out the externally applied field, at which time there is no net force acting on the charge and charge redistribution within the reticle stops. (This phenomenon whereby

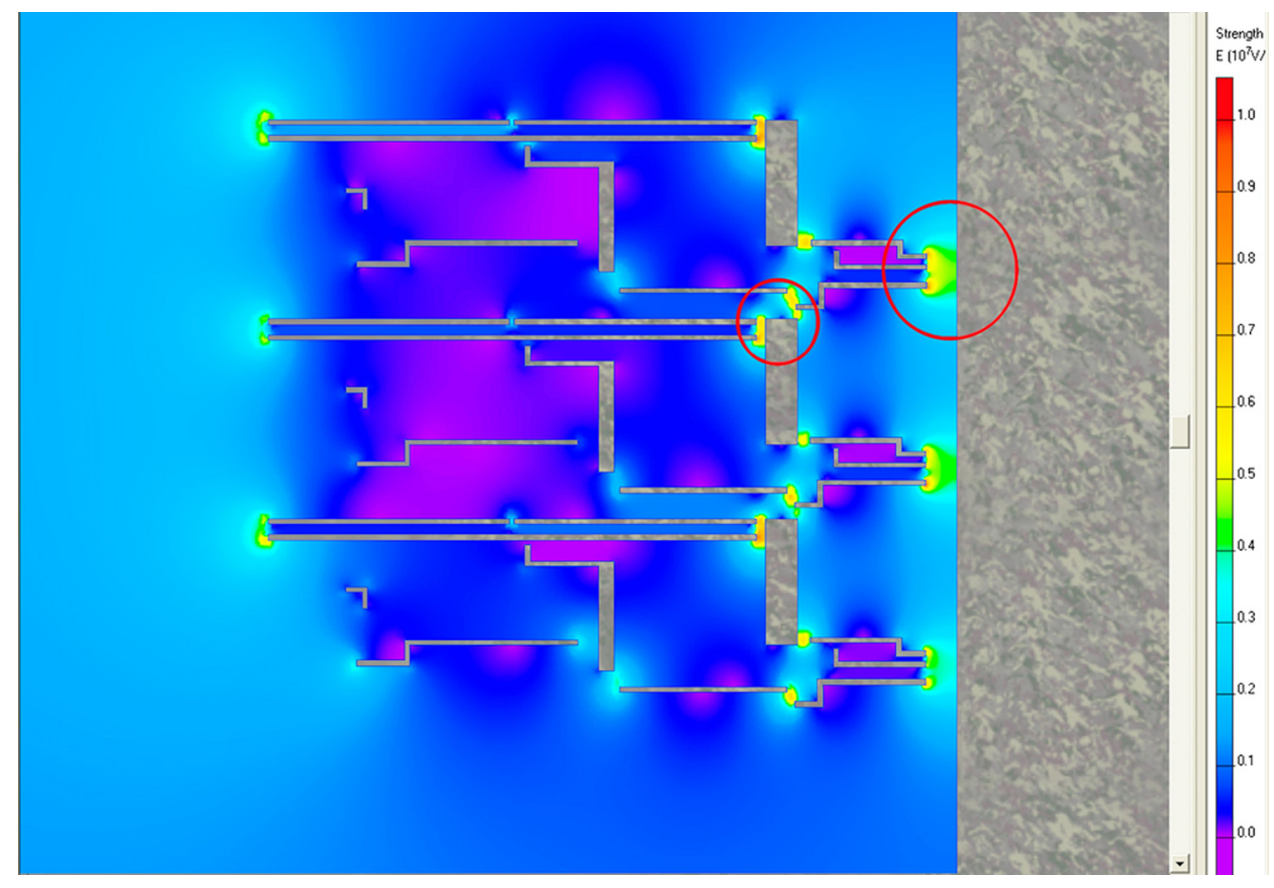

Fig. 1 Two-dimensional finite element simulation of the interaction of an electric field with the pattern area of a reticle. The gray structures represent isolated conductive lines, with the large gray block on the right representing the border around the image area. A uniform electric field is applied from left to right. Field strength is reduced in some areas and amplified at the ends of long lines, particularly between closely adjacent features near the edge of the image (circled). 
field-induced charge redistribution neutralizes an internal electric field is why it is often said that an electric field cannot exist within a conductor. It can, but the mobility of the electrons in a conductor is so high that the resulting charge redistribution cancels any internal field almost instantaneously. The correct statement is to say that an electric field cannot exist within a conductor at equilibrium).

Hence, upon exposure to an electric field, a reticle will quite quickly reach a new state of equilibrium in which there is no internal electric field present. All field-induced damage occurs while a net electric field is present within the reticle and the reticle is re-establishing a new equilibrium through charge displacement, so damage can be caused every time the field conditions around the reticle change and the reticle reacts to the disturbance of its equilibrium. Since a reticle reacts so rapidly to changes in its electrical environment, even very short duration field perturbations can be highly significant, as explained later.

\section{How Electric Field Damages a Reticle}

Field induction is a nonlinear process, and the computer simulation data of Fig. 2 show how the field induction in a reticle changes as the separation of conductive features is varied in a constant external electric field. The trend with decreasing device geometries and increased densification of reticle structures is toward the induction of lower potential differences between adjacent features in a reticle pattern, while the local (i.e., internal) electric field strength between those features rapidly increases. This radically changes the nature of any damage that may be caused by the field induction.

There are several ways in which field induction can cause damage in a reticle, with the damage either being dependent on the voltage or the electric field strength between features, according to the different physical processes involved in each damage mechanism.

The most familiar damage mechanism that occurs under the strongest field induction is an ESD event, which happens when the charge that is being forced to move within the reticle by the electric field suddenly jumps across the gap between conductive features as a spark. Field emission of electrons from the sharp edges of the reticle features

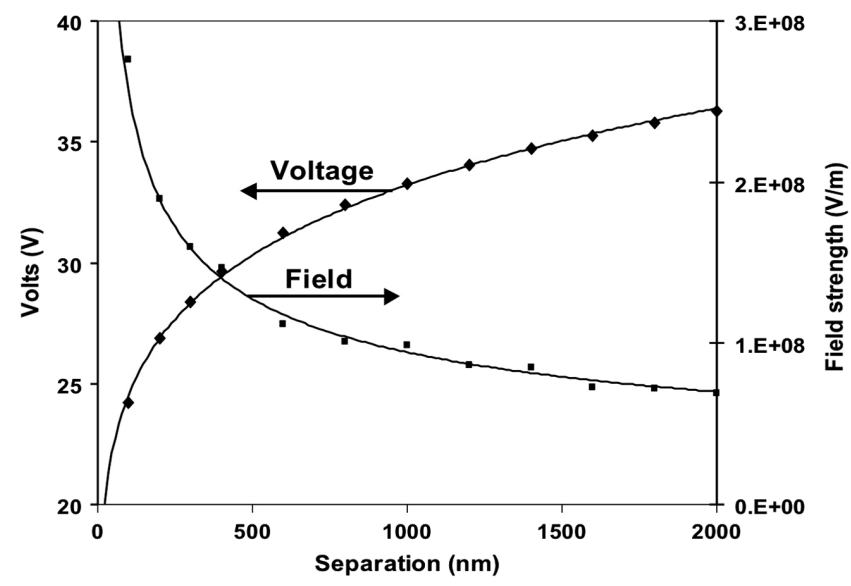

Fig. 2 Two-dimensional finite element analysis simulation of the induced potential difference and field strength between two isolated conductive lines as a function of their separation in a constant external electric field. under the influence of the locally amplified electric field (as shown in Fig. 1) leads to a cascade of ionization of the air molecules in the gaps between the features. This temporarily breaks down the air's electrical resistance, effectively creating a "short circuit" that allows a very high current to flow through the air for a very short time (nanoseconds).

The air discharge process is dependent on sufficient voltage being induced between conductors, this voltage being a multiple of the ionization potentials of the air molecules. For example, in a reticle with $1 \mu \mathrm{m}$ feature spacing, the voltage needed to generate a spark (the "ESD threshold") has been measured to be about $150 \mathrm{~V} .^{11}$ The power dissipated by the spark can be sufficient to vaporize the conductive material in the reticle pattern, as shown in Fig. 3.

ESD prevention technology is generally designed to keep the amount of any field induction below this ESD threshold. But regardless of whether the absence of a significant amount of ESD damage in production reticles today is due to the adoption of these ESD countermeasures or is due to the geometry-dependent change in field induction shown in Fig. 2, any remaining electrostatic stress experienced by the reticle can continue to induce other forms of damage. ESD suppression alone is not sufficient to prevent electrostatic damage in a reticle.

In addition to charge moving by conduction of electrons through the air as a result of field emission, it is also able to move across the surface of a reticle forming a "leakage current." This surface leakage current is often referred to as "charge dissipation" and it can be enhanced by humidity, since the adsorption of water molecules onto an insulating material generally reduces the surface's electrical resistance. However, in semiconductor factories the air humidity is kept quite low, which means that such charge dissipation due to humidity is reduced (this is why static charge is such a problem in semiconductor factories). Furthermore, in factories where $193 \mathrm{~nm}$ lithography is used, reticles are often stored in extremely dry conditions to suppress haze formation. This also suppresses charge dissipation. Since the surface resistivity of a reticle is quite high, any surface current will be small and the time scale for the charge displacement process will be long, so the power of any natural charge dissipation that

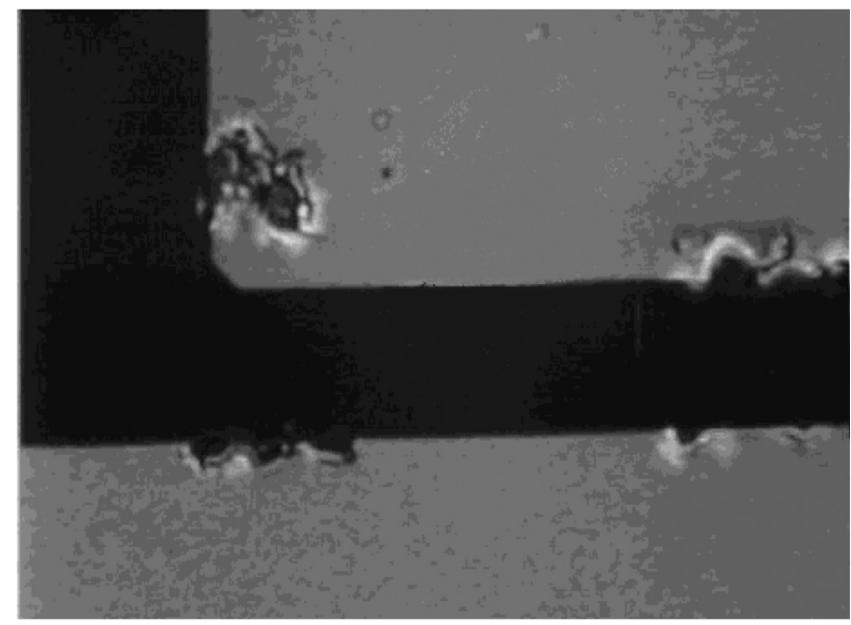

Fig. 3 The typical appearance of ESD damage in a reticle; parts of the conductive film on opposite sides of a gap between features may be vaporized where sparks have jumped across the gap. 

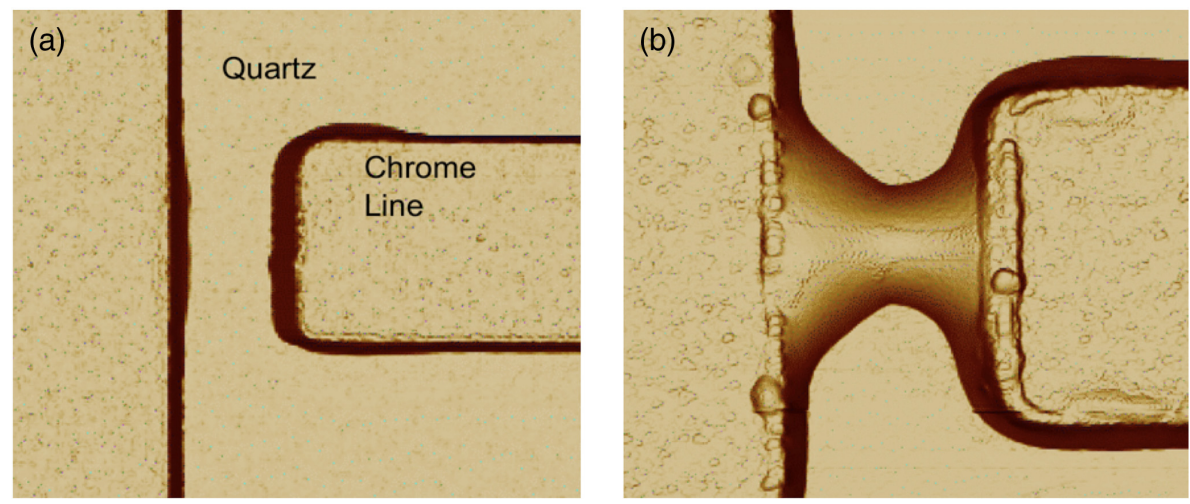

Fig. 4 EFM damage induced in a test reticle by applying calibrated electric fields: (a) the onset of degradation by EFM type 1, with a meniscus forming at the base of the chrome line (appearing as a widening of the dark edge of the feature). (b) Complete bridging of the gap between features by chromium that has migrated through EFM type 2. (Atomic force microscope images courtesy of International Sematech).

takes place is low and it does not result in ablative damage as is seen with ESD.

However, electrons are not the only mobile charge carriers present on the surface of a reticle; the chromium atoms in chrome-on-quartz reticles can carry positive charge. Under the influence of sufficiently strong electric field, chromium ions can be produced at the edges of the conductive features and these can move across the surface of the reticle in a process called electric field-induced migration (EFM type 2$),{ }^{7-10}$ as shown in Fig. 4(b). This process also dissipates very little power, but it is not the power of the event that causes the damage to the reticle as it is with ESD, it is the physical displacement of the chromium, which happens gradually and cumulatively, atom by atom.

As the level of field induction in a reticle falls below the threshold for the generation of chromium ions, another damage mechanism called EFM type 1 takes place as shown in Fig. 4(a), which is attributed to the field-enhanced thermal diffusion of neutral chromium atoms. It causes a meniscus to form at the base of the chrome features, with chromium atoms also diffusing out onto the quartz surface and forming an almost imperceptible film. The thin film of chromium oxide thus formed reduces the light transmission in the clear areas of the reticle that are most affected by EFM, causing across-chip line-width variation (ACLV) in the printed image. ${ }^{12}$ EFM type 1 is similar to the reticle degradation process that takes place under $193 \mathrm{~nm}$ UV irradiation in a lithography tool, which is known as the Sun Effect. ${ }^{13,14}$ In EFM type 1, the thermal diffusion is enhanced by the presence of an electric field, while in the Sun Effect it is enhanced by the absorption of $193 \mathrm{~nm}$ UV photons.

More detailed descriptions of all of the characteristics of EFM degradation and the experimental quantification of it have been published previously. ${ }^{9,10}$

It should be noted that EFM is dependent on the local field strength, not the induced voltage between features. However, to make a simplistic comparison between the level of field induction that is needed to produce ESD and that which causes EFM, in modern production reticles with feature separation measured in nanometers, EFM would start with an induced potential difference of very much less than one volt. This is illustrated by the computer simulation of Fig. 5, which represents a cross-sectional view of field induction in a metal-on-quartz reticle with an absorber thickness of

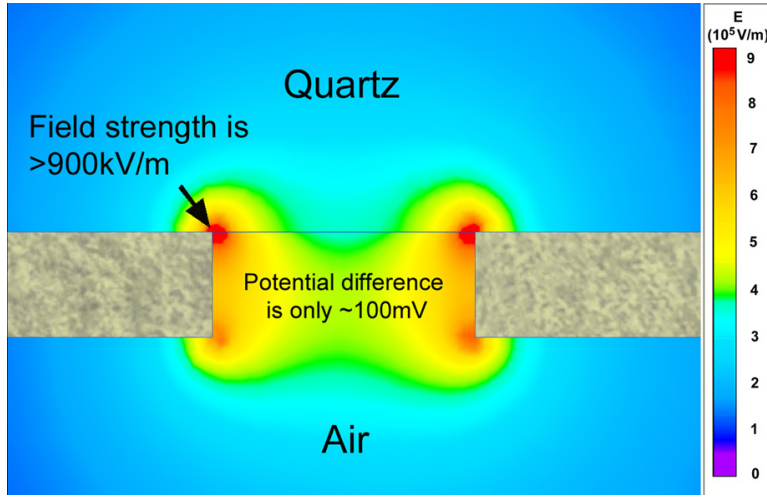

Fig. 5 Computer simulation of field induction in a metal-on-glass reticle with a feature separation of $250 \mathrm{~nm}$. The concentration of the field at the edge of the line in contact with the quartz substrate produces field strength above $900 \mathrm{kV} . \mathrm{m}^{-1}$ with a potential difference of only around $100 \mathrm{mV}$ induced between the features.

$100 \mathrm{~nm}$. In the simulation, an electric field of $70 \mathrm{~V} / \mathrm{cm}$ is first generated (equivalent to the ITRS maximum field guidance for the $65 \mathrm{~nm}$ technology node) then the reticle model is inserted into the field. The reticle structure is represented by a block of glass with two conductive lines on the lower surface with their ends facing each other at a separation of $250 \mathrm{~nm}$, each line being $25 \mu \mathrm{m}$ long. The top half of the cross-sectional view is the quartz of the reticle, and the bottom half shows the underside of the reticle with the metal lines on the surface. It is seen that a very high local field strength is generated at the boundary between the metal line and the quartz, even though the potential difference induced between the features is only around $100 \mathrm{mV}$ (approximately three orders of magnitude below the ESD threshold but very close to the experimentally determined onset threshold for EFM type $1^{10}$ ).

In addition to the inherent amplification of electric field by the reticle structure as shown in Figs. 1 and 5, EFM further amplifies the field concentration by the reticle by creating a sharp meniscus at the boundary between the metal and the quartz substrate. Atomic force microscope measurements of the edge profile of reticle features that have been degraded under calibrated electrostatic stress conditions, as shown in 


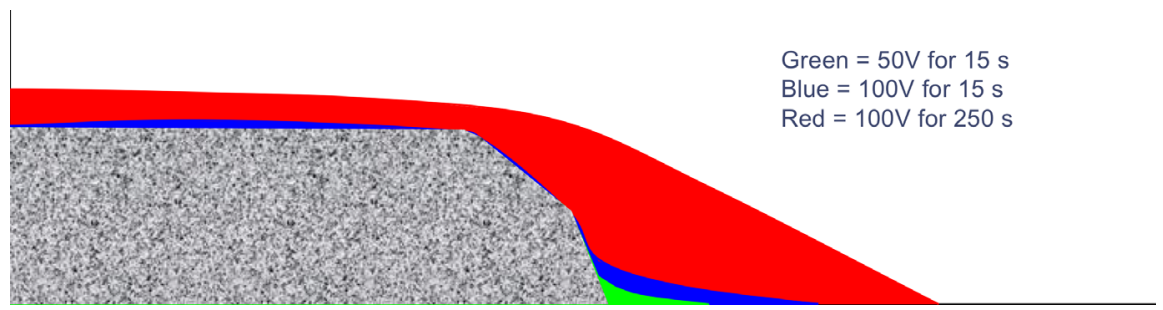

Fig. 6 Atomic force microscope line-edge profiles of reticle features with different stages of development of EFM type 1 and EFM type 2, shown approximately to scale. (Data obtained during the experimentation described in Ref. 9). The gray block is the original line shape.

Fig. 6, reveal the stages of EFM degradation that could ultimately take place in a reticle exposed to electric field.

The first sign of field-induced degradation in the reticle is the formation by EFM type 1 of a sharp meniscus at the base of the metal line, as indicated by the green AFM line-scan. This meniscus has an extremely shallow contact angle with the quartz substrate of $<10$ degrees. Double the electrostatic stress applied for the same amount of time causes the meniscus to grow more rapidly, and shape changes also start to take place on the body of the reticle feature, as shown by the blue AFM line-scan. With the high level of electrostatic stress applied for a longer time, EFM type 2 dominates as shown by the red AFM line-scan. Significant deformation of the reticle feature is caused, which ultimately would lead to gap bridging of the kind shown in Fig. 4(b).

The shallower the contact angle is between the metal and the quartz substrate and the more the reticle degradation progresses, the greater will be the field strength amplification at that point in the reticle. This is illustrated by the computer simulations of Fig. 7, which represent field induction in a reticle gap "as manufactured" and in the same gap with the same applied "external" field after degradation by EFM to the extent shown by the red AFM line-scan of Fig. 6.

It can be seen from these simulations that, as well as being a cumulative damage mechanism, EFM is actually selfenhancing. A reticle may begin its working life with fairly good resistance to field-induced damage, but as degradation inexorably progresses the reticle will become increasingly sensitive to electric fields and its deterioration will accelerate, unless its exposure to electric field is prevented.

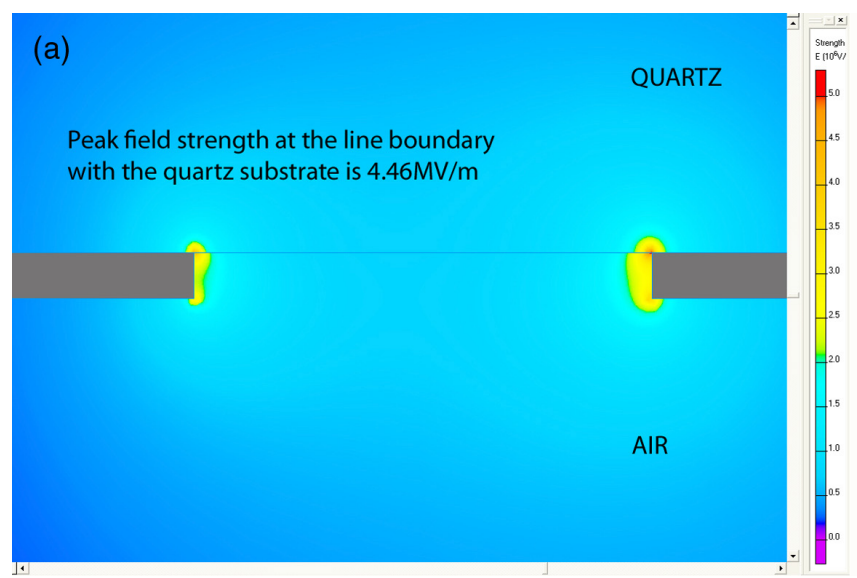

It is important to appreciate that the atomic force microscope images of Fig. 4 have a much higher resolution than images obtained with the type of optical reticle inspection systems that are used in semiconductor manufacturing facilities. So, while the nature of field-induced reticle degradation can be observed very clearly under laboratory conditions, it is not possible to see such detail with a normal optical reticle inspection tool. This is one reason that EFM is so difficult to identify in a semiconductor manufacturing environment.

Another reason for failing to identify EFM is that an affected reticle would probably start to print defective wafers long before the degradation had reached such an advanced stage of development, as shown in the paper by Rudack et al. ${ }^{15}$ Hence, the reticle would probably be sent for cleaning in the hope that this would rectify the problem. This characteristic has been confirmed in a semiconductor production fab, where yield loss was suffered despite the regular reticle inspections in the fab having detected no significant reticle defects. ${ }^{16}$ The problem was subsequently attributed to EFM following detailed (and destructive) failure analysis of the affected reticle, which detected migrated chrome.

This emphasizes the difficulty of detecting and correctly identifying the kind of cumulative reticle damage that now occurs as a result of electric field exposure. Since the degradation of the reticle takes place over an extended period, unlike with ESD which tends to be instantaneous and catastrophic, EFM is a pernicious damage mechanism. It would be impossible to identify all the areas in a production environment where electrostatic stress had been produced that had contributed to the degradation of the reticle. Levels of

Fig. 7 Finite element analysis of the field strength induced between adjacent reticle features by a fixed applied electric field at different stages of reticle degradation by EFM. (a) The maximum induced field strength in the "as manufactured" reticle with nominally vertical side walls. (b) The local field strength after the reticle feature has been degraded by EFM as shown in Fig. 6 (corresponding to the red line-scan). 


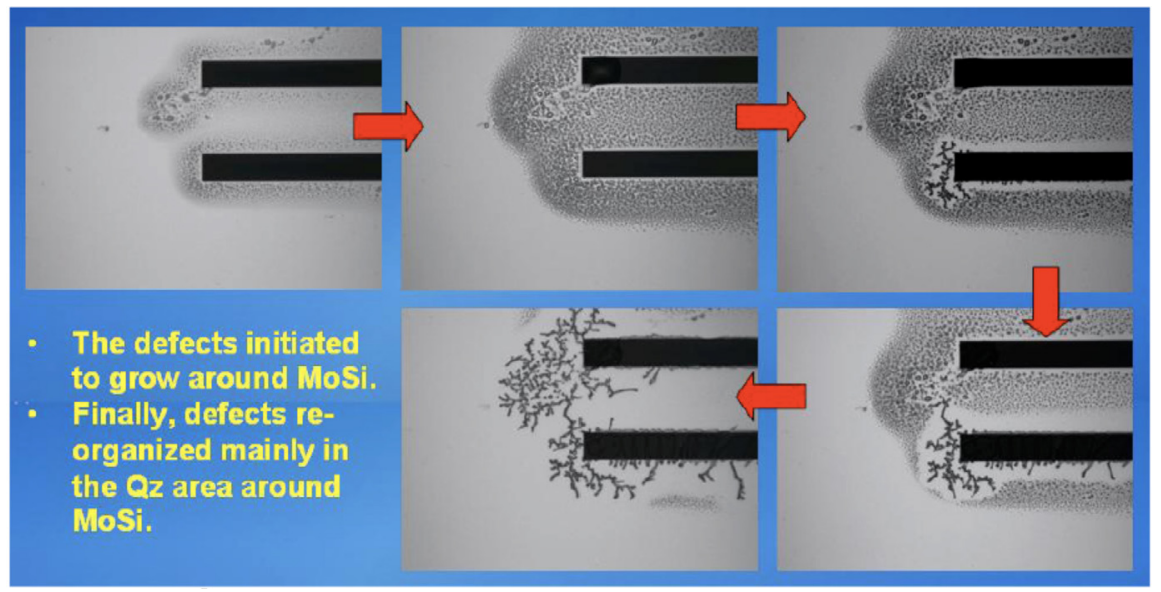

Fig. 8 The progression of haze formation in a MoSi reticle. ${ }^{17}$ Haze growth is seen to be enhanced around the tips of the lines, which is where electric field strength would be concentrated if an electric field should penetrate the reticle.

exposure to electric field that would be totally insignificant in terms of ESD risk become highly significant when considering the progressive degradation of a reticle by EFM.

Yet degradation through EFM is not the only way in which electric field passing through a reticle might cause yield loss. In the past decade, haze formation has become a serious problem. The development of haze on the reticle is primarily a result of exposure to $193 \mathrm{~nm}$ wavelength UV light in the lithography tool, which causes ionic contaminants adsorbed on the surface of the reticle to react and form opaque crystals. However, Ben Zvi et al. ${ }^{16,17}$ reported that haze growth can be stronger in the areas of the mask with denser features than in relatively open areas, which suggests that either the features themselves are the source of something that causes haze or the features are affecting the growth of haze in some other way. They also report that there is a form of transmission loss that develops most strongly around the periphery of the image area, which coincidentally is the region of the reticle where field induction is strongest. ${ }^{2}$ This would cause ACLV in a similar way to the Sun Effect, ${ }^{13}$ but with the opposite radial characteristic.

A conclusion that follows from these characteristics is that electric field could be a contributory factor in haze formation, and evidence to support this deduction can be seen in the images of haze growth in the MoSi mask in Fig. 8, reproduced from Ref. 17. These images show that the haze crystal nucleation is enhanced at the ends of the conductive features where the local electric field would be the strongest, closely matching the field strength simulations of Fig. 1. This is a plausible interpretation because for haze crystallites to form, contaminant ions of opposite polarity that are weakly adsorbed on the reticle surface need to come together and react to form a solid compound. An electric field would induce migration of these weakly adsorbed ionic species, which would move across the reticle surface in opposite directions following the electric field lines. This field-induced migration would enhance the probability that the different contaminants would encounter each other on the surface and then be able to react to form haze crystallites.

When a reticle experiences a change in its internal electric field, charge displacement takes place to negate it, so ESD countermeasures that may succeed in suppressing ESD but which are not completely effective at preventing electric field from reaching a reticle increase the likelihood that any electrostatic degradation of the reticle will be cumulative in nature. Every time the reticle's internal field conditions change, further degradation may occur; hence, it is essential when assessing the electrostatic risk to consider the temporal aspects of a reticle's interactions with electric field as well as the field strength that might reach the reticle. This can be visualized with the aid of the schematic diagrams in Fig. 9.

If a constant electric field is applied as in (a), there is one displacement of charge within the reticle and the amount of EFM that may be caused is related to the amount of charge that has been displaced. If as in case (b) the field is applied to the reticle and after a time removed again, charge is first displaced as in (a); then, when the field is removed the displaced

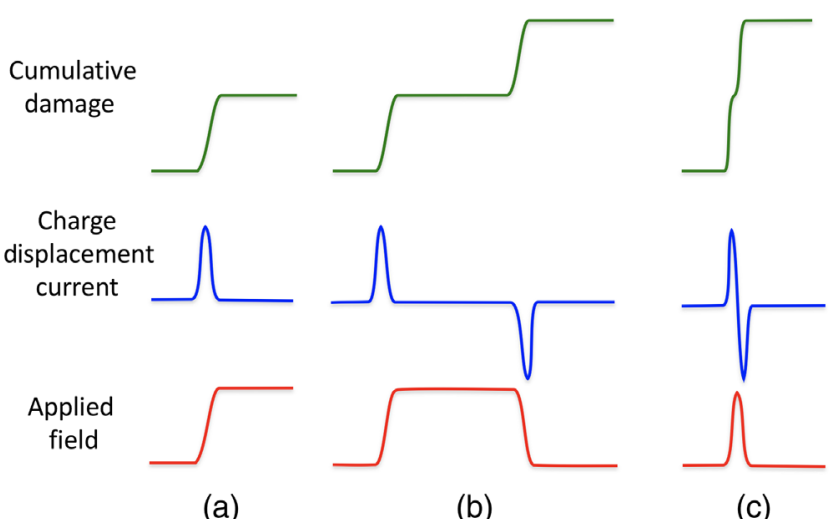

Fig. 9 Schematic representation of how exposing a reticle to an electric field induces charge migration and with it, EFM: (a) charge displacement as a reticle is first exposed to a constant electric field; current flows until the charge displacement cancels out the internal electric field within the reticle. One step of EFM damage. (b) Charge displacement current flows as in (a) when field is applied, then when it is removed again the current flows in the opposite direction as the displaced charge returns to its original location. Two steps of EFM damage. (c) A transient field passes through the reticle; charge is rapidly displaced and then as the field collapses the current immediately flows back in the reverse direction, causing the same amount of EFM as in case (b). 
charge returns to its original location, causing a current to flow in the opposite direction to the first displacement current. So, there are two charge displacement events in case (b) and twice as much EFM degradation would be caused as in case (a). If a short duration field transient is applied as in (c), the reticle reacts rapidly to the changing field and about the same amount of EFM will be caused as in case (b), but on a very much shorter timescale. Every time the field direction or its magnitude changes, the result will be approximately as shown by these simplified schematics.

\section{Consequences of Field Induced Reticle Damage}

One should not make the mistake of believing that because EFM is a less spectacular form of damage than ESD that it is less significant and can be ignored. The economic impact of EFM degradation in semiconductor production reticles is actually much greater than that resulting from ESD damage. When ESD damage occurs, a reticle may go instantaneously from producing good wafers to producing bad ones. The printed defect is normally detected quite promptly through routine wafer inspections and the affected lots of wafers can usually be reprocessed, minimizing waste. So, the cost impact of ESD damage to a reticle in a well-monitored production environment typically may be limited to tens of thousands of dollars, plus the cost of replacing the reticle.

However, with EFM damage, there is no instantaneous change to detect. For a long period of time an affected reticle will be printing sub-optimum wafers. The deviation in the printed pattern at wafer level may be detected through routine wafer inspections and the processing parameters may even be adjusted to try to compensate for it, ${ }^{17}$ but usually the production line will not be halted. The affected wafers will move on to undergo further processing steps, and they will pass beyond the point where they can be reprocessed. The production line thereby becomes filled with increasingly substandard wafers; although they may not necessarily cause devices to fail at final test, the printed defects could cause parametric variations in device operation and potentially reduce the final product's operational lifetime and/or reliability in the field.

If the reticle degradation is allowed to continue, the printed devices will have continuously degrading quality until at some point yield will begin to fall. When this happens, it may not be realized that a damaged reticle has been the cause of the problem. It can take a lot of time and detective work to diagnose the cause of such a failure (and time is money). ${ }^{18}$

If a degraded reticle is suspected, the cause of the degradation will probably not be known, in which case the reticle is usually sent back to the reticle maker for inspection and repair. The most commonly suspected cause of cumulative reticle degradation in modern semiconductor production is haze, so cleaning the reticle is normally the first thing to be tried. While some improvement may be achieved by removing any haze that is present, the cleaning process cannot selectively correct localized EFM degradation, so the reticle may still not pass quality control. At this point, a "failed clean" would probably be reported by the reticle manufacturer, and the reticle would simply be replaced with a new one. Consequently, EFM is generally not identified at this point because attention is usually focused on haze, so the staff members at the factory are likely to be completely unaware of any potential electrostatic contribution to the reticle's degradation.

Large numbers of wafers may have to be scrapped because of this kind of reticle damage, which means that the cost of a single case of EFM can be much more significant than a case of reticle ESD. A loss in excess of $\$ 1$ million, which was caused by repeated EFM damage to one particularly sensitive critical reticle, was reported in 2004. ${ }^{19}$

As reticle feature separations become smaller over time, smaller amounts of degradation will print a defective chip due to the nonlinearity of the lithography process and the increasing mask error enhancement factor. Resolution enhancement features that are used to correct printing nonlinearity are particularly susceptible to disturbance by EFM, owing to their extremely small separation from the primary reticle features and the criticality of their dimensions. Hence, it is becoming increasingly important to ensure that reticles are afforded appropriate protection against the effect of electric field exposure and the risk of EFM. Unfortunately, the kind of static dissipative plastic single-reticle pod that is increasingly being adopted by modern semiconductor factories does not provide adequate protection, and it actually accentuates the risk of EFM damage, as the following data show.

\section{Field Penetration into Reticle Pods}

It has been known ever since static dissipative reticle pods were introduced that they do not provide complete electrostatic protection for a reticle. Levit and Weil ${ }^{20}$ produced experimental data showing that an electric field applied to a static dissipative plastic reticle pod as a "step function" was transmitted to the interior of the pod as a pair of short-duration transient field spikes, being positive as the field increased and negative as it decreased. This is demonstrated in the recordings taken with the pod open and then with the pod closed, as shown in Fig. 10.

Figures 10(a) and 10(b) might look similar to the lower two schematics in Fig. 9(b), but they are not the same. Figure 9(b) represents what happens within a reticle when a step function field is applied, held constant for a period of time, and then removed, as would be the case with the reticle experiencing field exposure as in Fig. 10(a). Figure 10(b), however, shows how a field that is applied externally as a step function is converted by the static dissipative reticle pod into two internal field transients with opposite polarity. The static dissipative pod significantly modifies the field that reaches the reticle.

Hence, the static dissipative pod also significantly alters the damage that will be caused to the reticle: changing it from the situation of Fig. 9(b) into two field-transient exposure events with the capability of producing twice the damage of Fig. 9(c). It can be deduced from this that a static dissipative reticle pod may actually increase the risk of EFM damage by converting externally generated electric fields into a series of short-duration field transients that are transmitted to the reticle inside the pod.

At the time when Levit presented these findings to the Sematech ESD Symposium, reticle ESD damage was reaching epidemic proportions, so the urgent concern in the semiconductor industry was to reduce the damage rate by any means possible (EFM had not yet been discovered and 


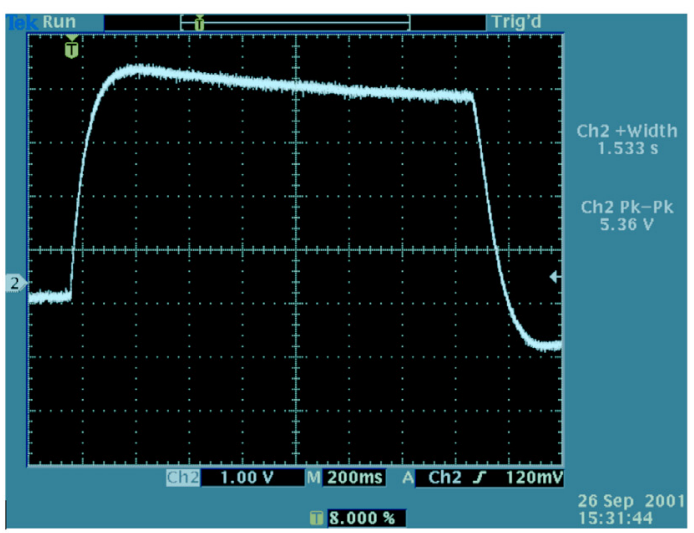

(a)

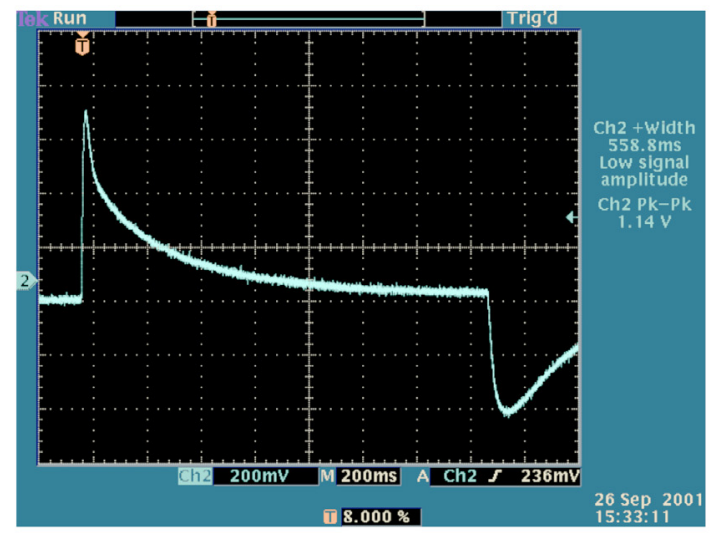

(b)

Fig. 10 Measurement by Levit and Weil of an applied electric field reaching the reticle position inside a static dissipative reticle pod. ${ }^{20}$ (a) Field profile measured with the pod open (no pod shell present between the high voltage electrode and the sensor). (b) Field measured inside the pod with the pod top in place.

the significance of field transients was not appreciated). Any reduction in the amount of electric field being generated by the pod itself (as measured using the methodology of the time, to be discussed later) was considered an improvement. So, even though it had been shown that the protection offered by a static dissipative pod was not perfect, the introduction of static dissipative single-reticle pods went ahead. Along with other static control measures being implemented at the same time, this significantly reduced the rate of reticle ESD damage in semiconductor factories, so static dissipative reticle pods have become widely adopted and are now the de facto standard in modern semiconductor production.

After EFM was discovered in 2003 and the significant risk that remains from reticle exposure to electric field below the ESD threshold was identified, ${ }^{7,21}$ fabs where electrostatic reticle damage was still taking place despite the adoption of static dissipative reticle pods carried out further investigation. In one such study, Helmholz and Lering at AMTC in Dresden measured field penetration into a variety of commercially available and prototype single-reticle SMIF pods to assess the relative field-shielding effectiveness of different static dissipative and "conductive" plastic reticle pods. ${ }^{4}$ They measured the field inside the pod directly with a sensor probe placed at the reticle position, which is similar to the method of Levit and Weil, and they also used a field-sensitive "Canary" test reticle as used in the earlier Sematech experiments. ${ }^{22}$

Their study showed that the more conductive the plastic, the greater the field attenuation that it achieved. (A similar observation was made by Turley et al. $^{23}$ ). However, even with the most conductive plastic pod available (a carbon nanotube-loaded PEEK prototype), Helmholz and Lering found a significant rate of field-induced ESD damage in the test reticle. If the pod could not attenuate electric field penetration into the pod sufficiently to prevent ESD damage in the test reticle, it certainly would not be capable of preventing EFM.

Both sets of authors documented the penetration of field transients into the pods they were testing, but nothing was said in their papers about this particular aspect of the field penetration; attention was only given to the relative magnitude of the field reaching the interior of each of the pods (as indicated by the sensors they were using - see later).
However, in view of the considerations presented here, showing that a risk of damage arises every time the field conditions within the reticle change, the temporal characteristics of the field penetration into a reticle pod are seen to be highly significant.

Levit and Weil's data showed that the field attenuation achieved by a static dissipative reticle pod rapidly decreases as the rate of change of the field increases. They reported a $20-\mathrm{dB}$ reduction in the field attenuation by the time the frequency of the stress had risen to just $25 \mathrm{~Hz}$, as shown in Fig. 11.

In 2000, Chubb had presented measurements of the electric field transmission characteristics of a variety of "ESD protective" packaging materials as a function of frequency over a much broader frequency range. ${ }^{24} \mathrm{He}$ identified that the standard methods for testing electrostatic protective packaging materials did not adequately consider the frequency (or rate of change, for a single isolated event) of the electrostatic stress, noting:

"Electrostatic spark discharges involve current rise times and voltage collapse times down to below $1 \mathrm{~ns}$. Lower voltages shorter times. Transport packaging hence needs to provide >200:1 attenuation for frequencies to $1 \mathrm{GHz} . "$

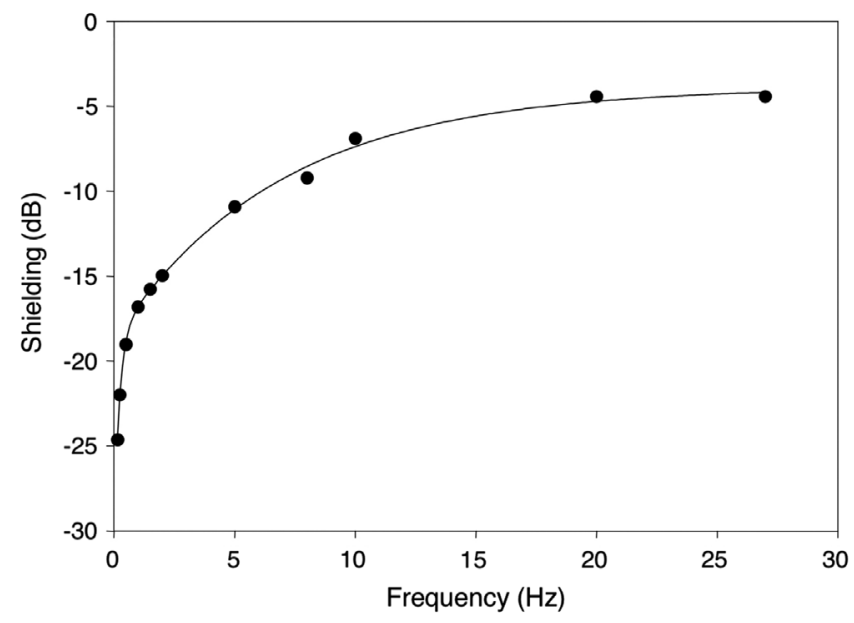

Fig. 11 Field attenuation by a static dissipative reticle pod as measured by Levit and Weil. ${ }^{20}$ 


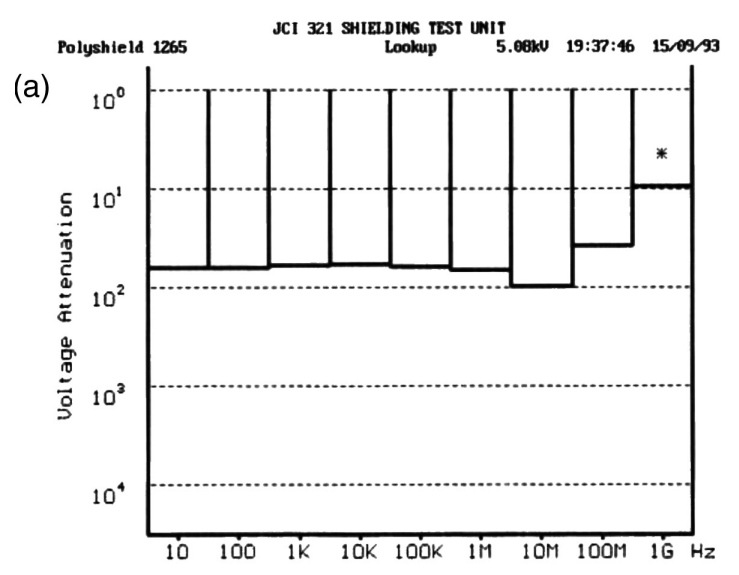

Shielding performance of met allised shielding bag

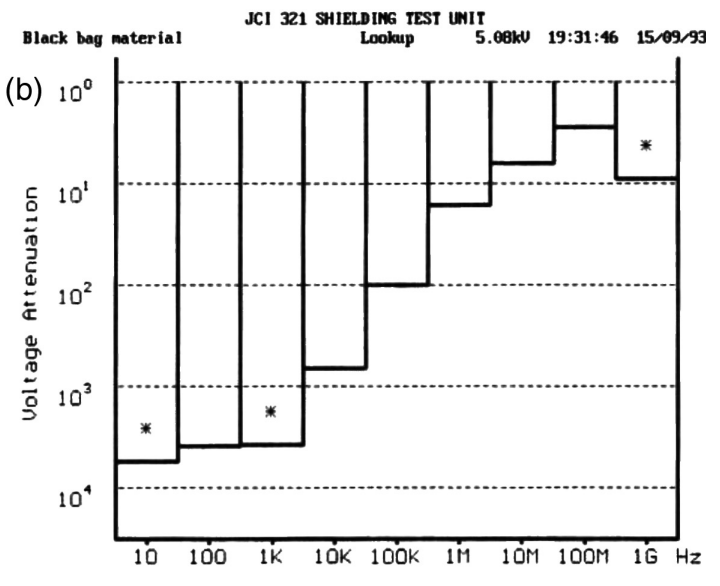

Shielding performance of carbon loaded 'black bag'

Fig. 12 Chubb's measurement of the field penetration characteristics of different materials used to make static protective bags: (a) field attenuation by a metalized plastic bag; (b) superior field attenuation at low frequency by carbon loaded (static dissipative) plastic bag, but inferior shielding at higher frequency.

It is important to understand that his specification related to the ESD protection of packaged semiconductor devices. Much better shielding than this is required for the protection of reticles, which are far more field sensitive and are damaged by much lower levels of field induction than packaged semiconductor devices. Chubb's study presented data showing that different ESD-protective materials have significantly different transmission characteristics as a function of frequency. The conductive plastic type of bag tends to suppress low frequency fields more effectively than metalized plastic bags, but they both allow high frequency signals capable of generating ESD to penetrate, as shown in Fig. 12.

The variable attenuation as a function of frequency reported by Levit and Weil and as shown in Fig. 12(b) is a characteristic of all static dissipative materials. Effectively they operate like a high-pass filter, blocking slow changes in the field quite effectively but providing less attenuation the faster the field changes. This characteristic is extremely significant when considering how materials have conventionally been tested and their suitability for making reticle pods has been evaluated.

The standard test for evaluating the electrostatic performance of a reticle pod for many years has been a "wipe test" in which the pod is rubbed vigorously with either a cloth or a clean-room glove to tribocharge it. A handheld field meter is then used to measure the field that the pod produces at a given distance, as described in SEMI Standard E43. If the field strength is below a prescribed level the material is considered safe to use, and if it is higher than the recommended maximum it is designated as unsuitable. But this test makes no measurement of the field inside the pod itself, and it is effectively a steady-state only test. This kind of test is completely inadequate for assessing the safety of a material for reticle pod construction, and it gives a false indication of the suitability of static dissipative plastics.

Static dissipative materials do not retain static charge on their surface for longer than a few seconds, so when they are tested with a wipe test they exhibit little or no persistent electric field (Helmholz and Lering observed that none of the pods they were testing exhibited tribocharging in a wipe test). Any electric field generated by static dissipative plastic rapidly disappears, giving a person conducting a wipe test the false impression that the material presents no electrostatic risk to a reticle. But all materials-including static dissipative plastics - are capable of being tribocharged, producing an electric field, and in the case of dissipative plastics, modifying the field that they transmit.

The more conductive the plastic is made, the more quickly the material responds to static charge generated on its surface or to an external field and negates it. However, such material will still allow fast field transients to penetrate a reticle pod and affect the reticle inside, as indicated by Figs. 10 and 12 . When this is taken into account, static dissipative plastic is seen to expose reticles to a significant electrostatic risk if it is used to make a reticle pod or box.

\section{Direct Measurement of Field Penetration into Reticle Pods}

Whenever the conditions around a reticle change-which can happen simply by moving nearby objects, parts of equipment or the reticle itself - the pattern of any electric field that is present within the reticle will be affected. When this happens and the field passing through the reticle alters, charge moves within the reticle to cancel out the change in its internal field. This can very easily cause EFM because the amount of field induction that causes EFM in a reticle is so low. If a reticle pod allows any electric field to reach the reticle-particularly if it allows it through low levels of rapidly changing electric field or field transients-the risk of EFM is significant.

The fact that static dissipative reticle pods can become tribocharged, can produce significant field transients, and do allow these transient fields to reach a reticle inside is demonstrated by the measurement in Fig. 13. This is a chart of the electric field recorded by a specially designed sensor reticle housed in a static dissipative single-reticle pod. The sensor reticle records the electric field that a normal reticle would experience under the same conditions. ${ }^{25}$ As the reticle is carried to a piece of reticle handling equipment, it experiences some electrostatic stresses as shown by the small peaks in the initial part of the chart. Unlocking the SMIF pod then generates two significant "spikes" of electric field that penetrate the reticle. These field transients are strong enough to cause EFM in field-sensitive production reticles. 


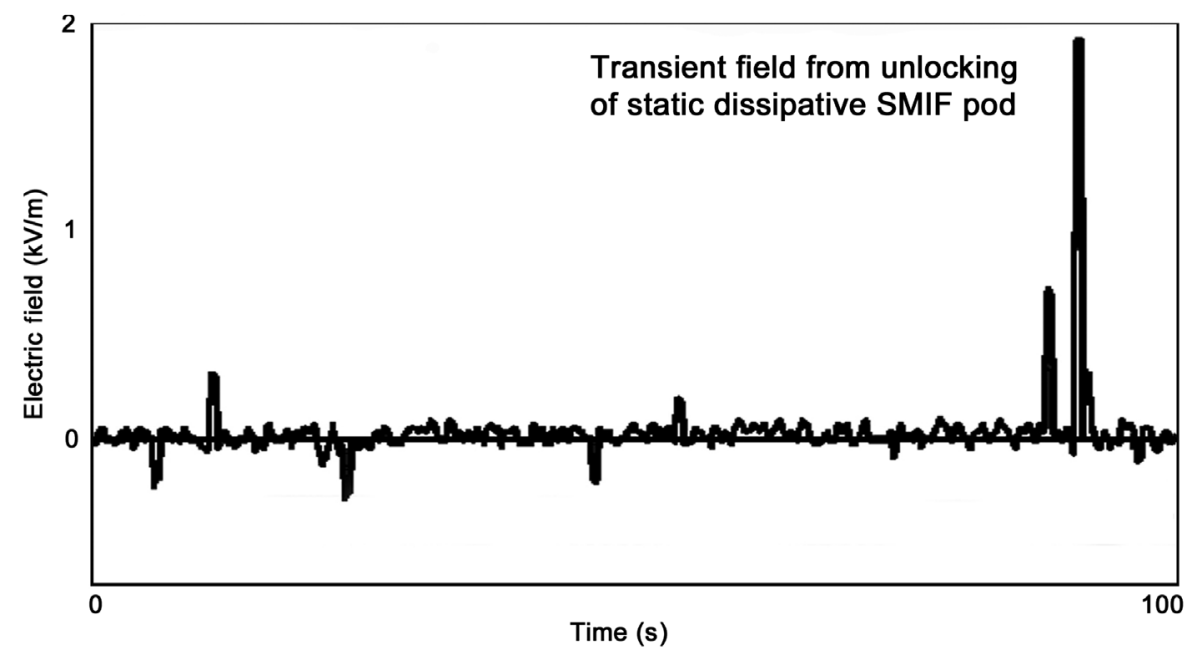

Fig. 13 Electric field recorded by a sensor reticle carried to a piece of equipment in a static dissipative single-reticle pod. Note the strong field transients caused by unlocking the static dissipative SMIF pod.

So, static dissipative plastic is prone to tribocharging, just like any other material. The strength and polarity of the tribocharging depend on the relative position in the triboelectric series of the two materials that contact each other, and the persistence of the tribocharging will depend on the conductivity of the material. In Fig. 13 it can be seen that the lock friction when opening the static dissipative SMIF pod generates two pulses of electric field. The field inside the pod rapidly rises and then decays immediately as current flows within the dissipative material to neutralize the static charge that has generated it, which is the risk scenario of Fig. 9(c). This demonstrates that the "high pass" transmission characteristics of a static dissipative reticle pod, as shown in Fig. 10 and predicted by the data of Figs. 11 and 12, are evident in real life situations. The pod itself actually generated the transient electric field in this case, during its normal use.

Figure 14 shows a recording of the electric field inside a typical static dissipative single-reticle pod, during handling in a semiconductor factory that was equipped with normal static-reduction measures, including ceiling mounted air ionizers. It can be seen that the handling of the pod repeatedly generates transient pulses of electric field that penetrate the reticle inside the pod. Such transients are unlikely to induce ESD in a production reticle unless it is one that is very sensitive to field induction, but EFM will almost certainly be taking place under these conditions. (These are believed to be the kind of conditions that were responsible for the reticle damage that caused \$1million worth of production losses in $2004 .^{19}$ The factory in that case was using the latest generation of static dissipative single reticle pods, had regular ESD audits, and had in place the most advanced ESD countermeasures available).

For comparison, data from the testing of a multi-reticle pod made from insulating plastic are presented in Fig. 15. The pod contains an all-metal cassette that provides partial shielding from electric fields. The sensor reticle was placed in the middle of the cassette, in a position that allows the greatest amount of field penetration through the open side of the cassette, as shown in previously reported computer simulation. $^{2}$ Three other reticles were placed in the slots below the sensor reticle to represent a partly loaded pod (The slowly undulating background field was caused by the accidental charging of these other reticles due to the workstation ionizer being switched off during the test).

It is seen that even under deliberately extreme test conditions that were designed to generate the maximum electrostatic stress, the field that reached the sensor reticle in the multi-reticle pod is comparable in intensity to that reaching a reticle carried in a standard static dissipative single-reticle pod inside a normal ESD-controlled

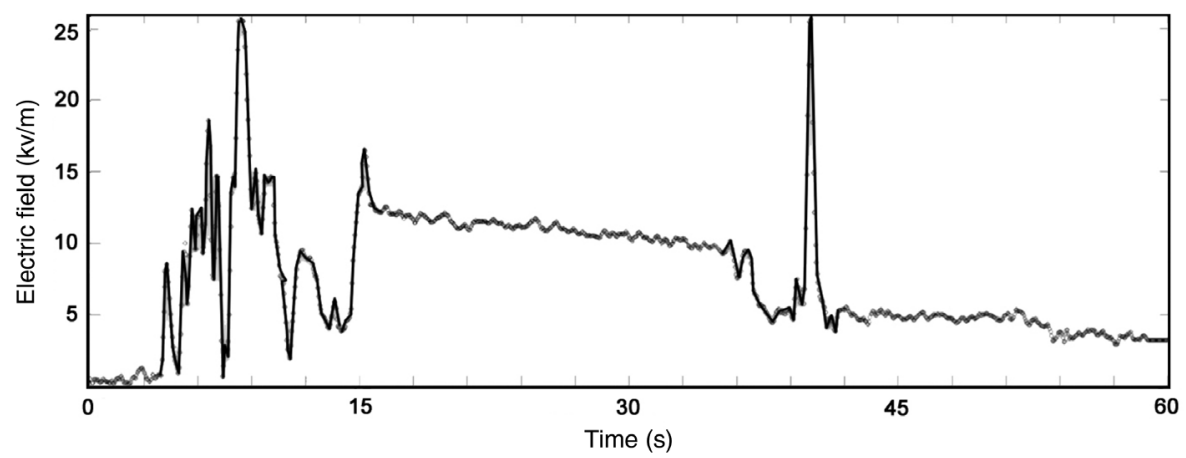

Fig. 14 Electric field recorded by a sensor reticle while being carried through a semiconductor production facility in a standard static dissipative single-reticle SMIF pod. Peak field strength recorded is $26 \mathrm{kV} \cdot \mathrm{m}^{-1}$. ITRS guidance for EFM prevention is $500 \mathrm{~V} \cdot \mathrm{m}^{-1}$. 


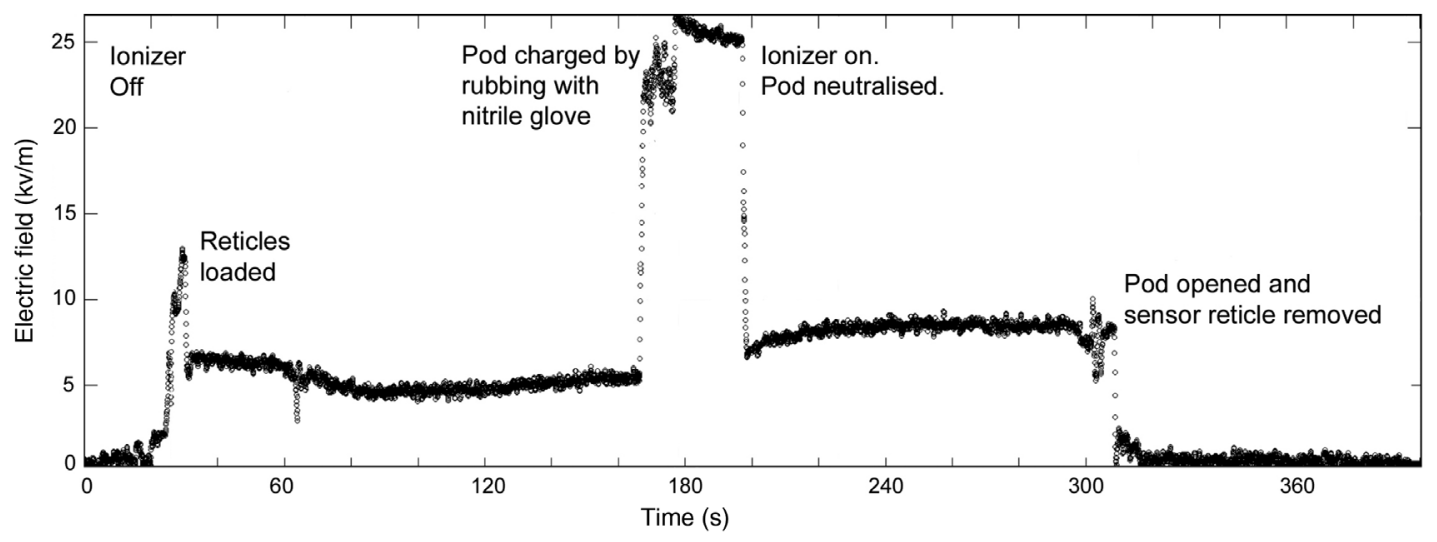

Fig. 15 Electric field recorded by a sensor reticle placed in the middle of an insulating multi-reticle pod with three other reticles below it. No ionization is operating when the pod is rubbed with a nitrile glove to tribocharge the side panel. The charge is subsequently removed using air ionization. Peak field strength recorded is $27 \mathrm{kV} \cdot \mathrm{m}^{-1}$ (The elevated background field was caused by accidental charging of the other reticles in the cassette during loading due to the workstation ionizer being switched off for the test-this field would normally be absent).

semiconductor production environment. The most notable difference, however, is the absence of field transients. Tribocharging of the insulating pod creates only one field perturbation event [as in Fig. 9(b)], rather than the multiple field transients recorded inside a static dissipative reticle pod (as in Fig 14). Hence, there is actually a reduced risk of EFM being caused by tribocharging of the insulating multi-reticle pod than there is by tribocharging of the static dissipative single-reticle pod. This experimental evidence is contrary to common perceptions about the relative risk posed by using static dissipative or insulating reticle pod materials, based on the "wipe test" approach and SEMI Standard E43.

Field penetration during normal multi-reticle pod use would be much less than in this deliberately extreme test since it is more usual that a multi-reticle pod would be fully populated. Under these circumstances, the reticles add to the shielding provided by the metal cassette and further reduce the amount of field penetration. This is demonstrated by the comparison shown in Fig. 16.

The first graph shows the field recorded at a central slot in the cassette without any other reticles present. This represents the "worst-case scenario" for field penetration. When the cassette is fully populated with reticles and the same stress is applied, the field penetration is reduced by about $80 \%$. With a fully populated multi-reticle cassette, the level of field that reached the test reticle even under deliberately aggressive conditions was considerably lower than that recorded inside a normal static dissipative single-reticle pod being carried in a normal semiconductor production environment. The field that would be likely to reach reticles carried in a full multi-reticle pod being handled with care in a normal ESD-controlled semiconductor production environment would be considerably lower than this and very much closer to the $500 \mathrm{~V} . \mathrm{m}^{-1}$ ITRS guidance for EFM prevention ${ }^{26}$ than is seen with the static dissipative singlereticle pod.

It is clear from the data that metallic shielding is the key to reticle preservation, providing protection that is fail-safe against any failure of ESD countermeasures or handling procedures anywhere in the handling environment. For this reason, a single-reticle SMIF pod with a fully metallic shell has been designed and tested. The all-metal shell forms

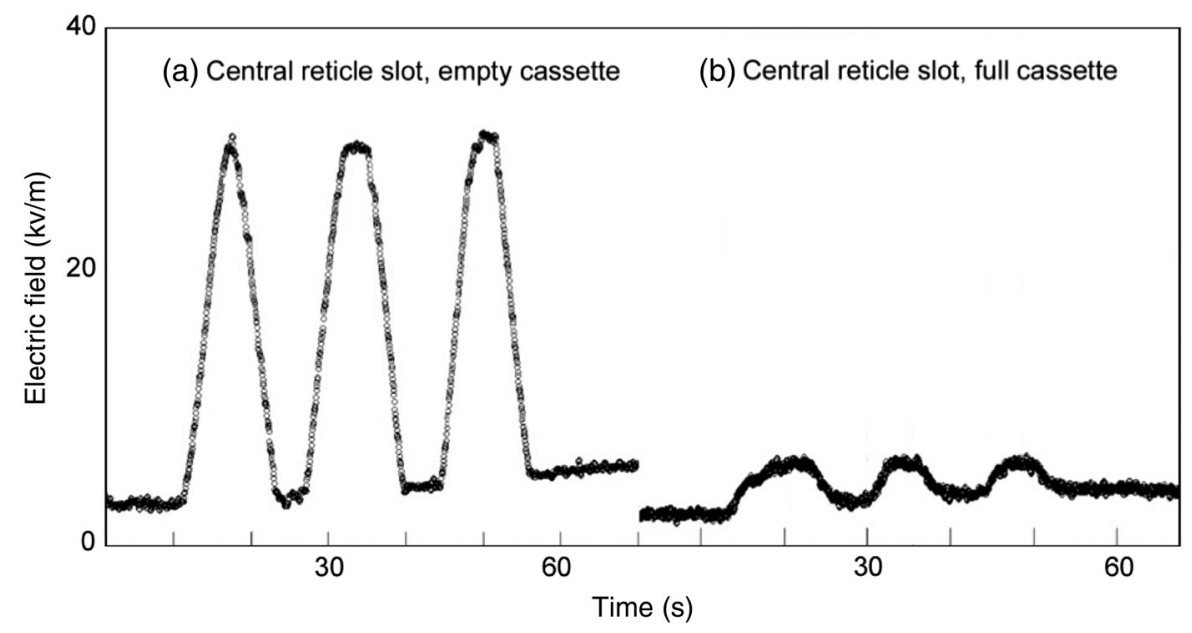

Fig. 16 Electric field penetration at a central position in a multi-reticle cassette (worst-case scenario for field penetration): (a) when the cassette is empty and (b) when it is fully populated with reticles. $13 \mathrm{kV}$ stress is applied using an external electrode, simulating tribocharging of the pod wall. Peak field recorded with the fully populated cassette is $7 \mathrm{kV} \cdot \mathrm{m}^{-1}$. 


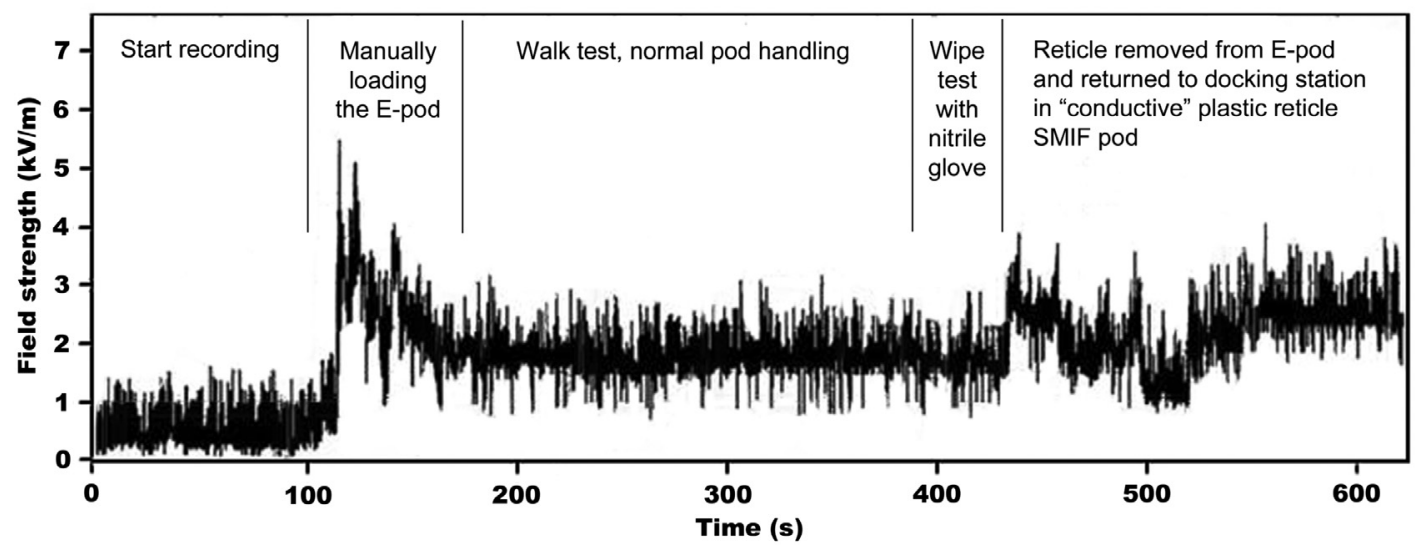

Fig. 17 Electric field penetration test of an all-metal single-reticle SMIF pod (E-pod) designed for protection against EFM. Noise in the trace is from the electronics and is due to the very low level of the signal.

a complete Faraday cage around the reticle, with the two separate parts of the pod shell being electrically connected to one another by sprung stainless steel electrodes as the pod locks are closed. The result from the electrostatic stress testing of this pod is shown in Fig. 17. (The noise is from the recording electronics, which are working at the limit of detection).

When the recording starts, there is no disturbance to the sensor reticle until it is manually removed from its conductive plastic single-reticle SMIF pod and transferred into the all-metal pod. Despite the reticle-handling environment being ionized through the use of ceiling mounted ionizers, the manual transfer step generates some significant field transients. The reticle also remains slightly charged after being loaded into the metal pod, as can be seen from the offset in the field readings after the transfer.

Once the sensor reticle is housed within the closed allmetal single-reticle SMIF pod, there is no deviation at all in the field reading, indicating that the reticle's electrostatic environment is completely stable-despite the attempt at the end of the test to tribocharge the pod by vigorously rubbing the insulating plastic pod handle with a nitrile glove. After this "wipe test" the sensor reticle was manually removed from the all-metal SMIF pod and returned to its docking station inside its conductive plastic single-reticle SMIF pod. The variability of the trace from the point of transfer into the conductive plastic SMIF pod up to the end of the recording indicates that the reticle's electrostatic environment inside the conductive plastic SMIF pod was not completely stable, showing that it was sensing the external environment.

The recent adoption of increasingly conductive plastic for reticle pod construction has reduced the ability of this sensor reticle to record field transients that may be transmitted to the reticle, because of the high-pass characteristics of such conductive plastic. The sensor reticle's recording electronics integrate for $50 \mathrm{~ms}$ per data point, so the recording device will progressively understate the magnitude of transmitted field transients as the "frequency" (rather the rate of rise and fall for a single field transient) increases above just $20 \mathrm{~Hz}$. Since more rapid field transient spikes and frequencies up to $1 \mathrm{GHz}$ and beyond are able to cause charge displacement and hence field-induced damage within a reticle, there is a significant part of the risk spectrum that cannot be detected by this sensor reticle.
As demonstrated during the testing carried out by Helmholz and Lering, even a picocoulombmeter connected to a fast storage oscilloscope did not detect the transient fields being transmitted by a carbon nanotube-loaded PEEK reticle pod. Yet the test reticle stressed inside the same pod suffered a significant amount of ESD damage, proving that dangerous field transients were indeed getting through to the reticle and were damaging it-even though they were undetectable by one of the most advanced electronic sensors available.

\section{Electrostatic Risks Outside Reticle Pods}

The primary focus of this paper so far has been on field penetration into reticle pods, mainly to try and counter the strongly established yet mistaken beliefs about the suitability of static dissipative plastics in reticle handling. However, there are many situations wherein a reticle in normal use is outside a reticle pod. The way the reticle is handled there will also affect the risk of electrostatic damage or cumulative degradation, so for completeness a few examples of some of these risk areas will now be given.

As shown in Fig. 18, the ionizers that have been introduced into most reticle handling and processing equipment to neutralize static charge are not necessarily benign. Pulsedfield ionizers can generate significant levels of electric field, so care must be taken to locate them where this field cannot reach a reticle. In this example, the ionizer is much too close to the reticle's handling path and every pulse of the field from the emitter tip will potentially have the capability to cause the EFM damage profile shown in Fig. 9(c) (or to promote other kinds of field-enhanced reticle degradation as suggested by Fig. 8). A similar case of a badly sited ionizer inside equipment was observed by Turley et al. ${ }^{23}$

Another problem exists as a result of the mistaken belief that grounding a reticle through static dissipative contacts helps to reduce the electrostatic risk by slowly draining away static charge to ground. The field recording presented in Fig. 19 shows the consequences of a reticle being introduced into a piece of processing equipment fitted with a badly maintained ionizer. The ionizer actually adds charge to the reticle which, being mainly insulating, cannot be neutralized by grounding. When the reticle is subsequently picked up and moved, the proximity of the grounded robot gripper strongly perturbs the electric field conditions 


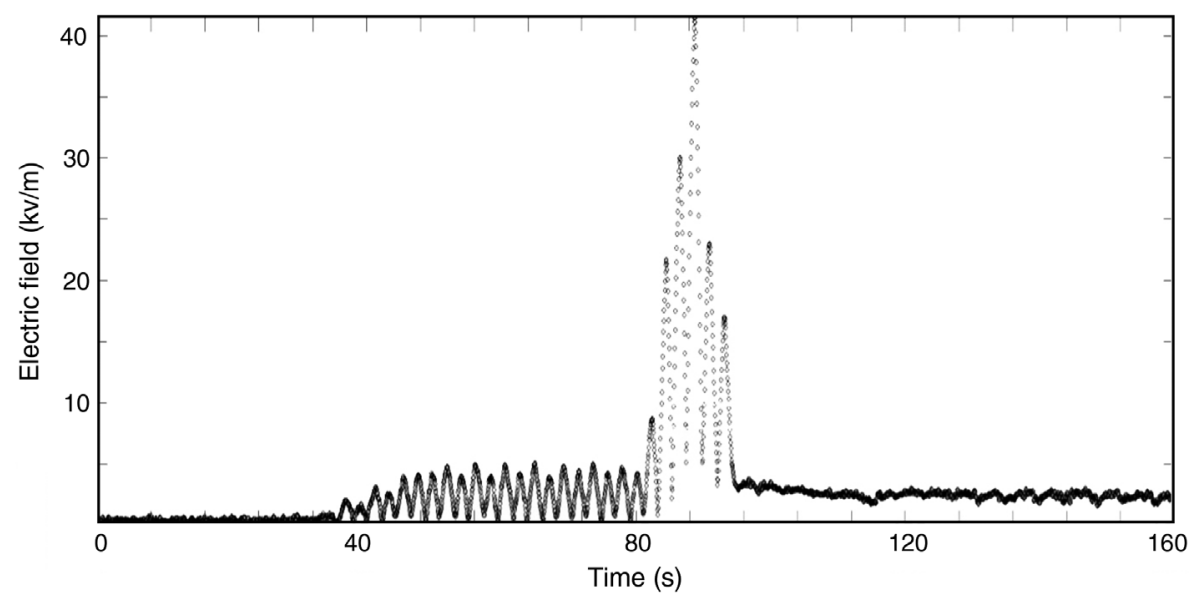

Fig. 18 Electric field recorded by a sensor reticle introduced to a piece of handling equipment fitted with an ionizer that is too close to the reticle handling path. As the reticle passes by the ionizer, it experiences a rapidly oscillating field, each transient of which is capable of causing EFM degradation of the reticle.

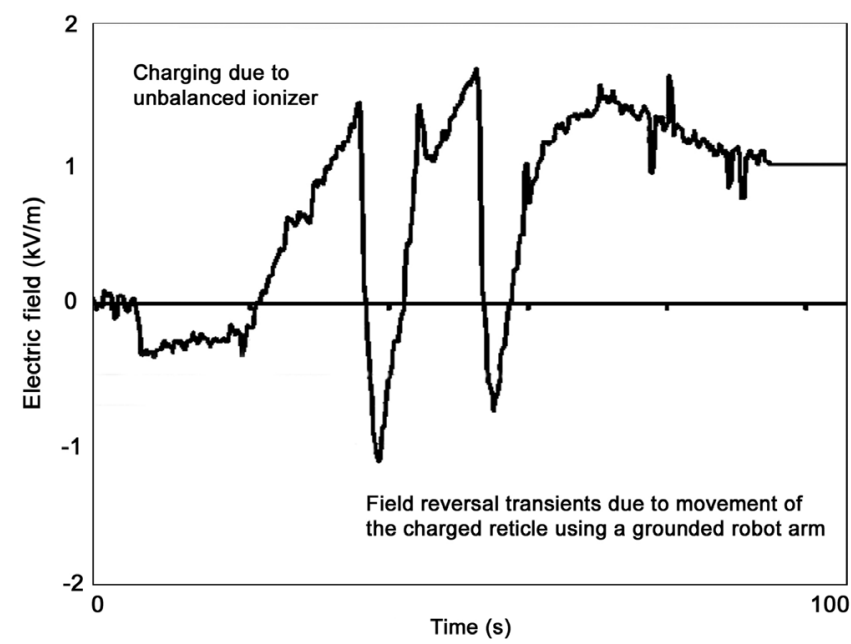

Fig. 19 Electric field recorded by a sensor reticle introduced to a piece of handling equipment fitted with an unbalanced ionizer and a grounded reticle handling robot with static dissipative reticle contacts.

and causes two field reversal transients within the reticle. Note that after the reticle has been moved each time there has been no reduction in the level of charge on the reticlethe use of static dissipative contacts and a grounded handling tool has not neutralized the reticle. The only impact that this equipment's reticle handler design has on the reticle is to greatly worsen the risk created by the ionizer being out of balance. Similar observations were again made by Turley et al. $^{23}$ in their reticle handling study, noting "The major spikes were caused by mechanical handling such as end effector picking up mask."

The same problem would exist in any situation where the handling or processing of the reticle caused it to become charged and the means used to move the reticle were metallic and connected to ground. One such example is reticle cleaning, as shown in Fig. 20. Here the cleaning process unavoidably causes the reticle to charge. The field strength within the reticle would be minimized during such a procedure by keeping any grounded equipment panels and internal apparatus as far as possible from the reticle. Neutralization using air

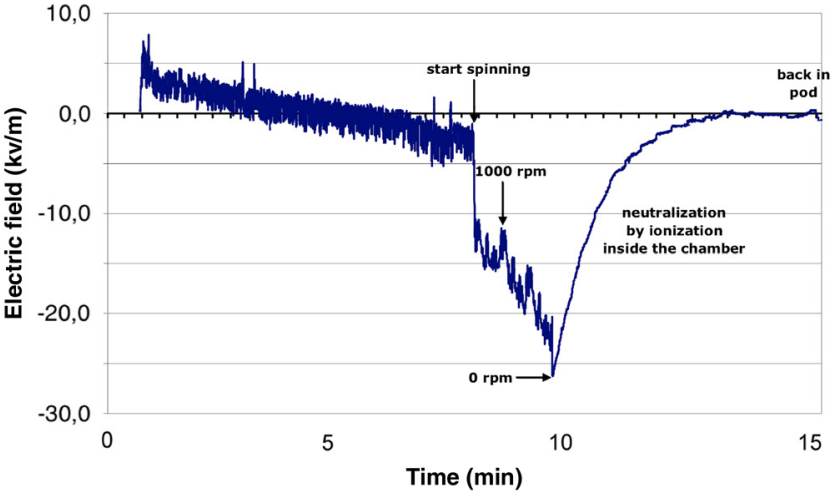

Fig. 20 Electric field recorded during reticle washing with deionized water followed by spin drying. Ionization to neutralize the reticle is essential after such a process, to avoid the scenario of Fig. 19 occurring when the reticle is being removed.

ionization is seen to be essential after this process, and sufficient time must be allowed for the ionizer to completely neutralize the reticle before it is collected by any grounded robot arm; otherwise, the scenario of Fig. 19 could be replicated. The problem of field perturbation by a grounded robot arm would be avoided if the arm were instead made of fieldtransparent insulating material, such as the kind of ceramic that is used in some wafer processing equipment.

\section{Discussion}

Early in the nineteenth century, Horatio Nelson of the British Navy was leading his ships in battle against the port of Copenhagen, which was in allegiance with Britain's enemy at that time, the French emperor Napoleon Bonaparte. The commander of the British fleet believed that the action was failing and ordered Nelson to retreat using signal flags. Not wishing to be deflected from his chosen course of action, Nelson, who had lost the sight in one eye during a previous battle, raised his telescope to his blind eye and pronounced, "I really do not see the signal."

This anecdote gives rise to the English language idiom of "turning a blind eye," which is used when someone deliberately ignores something that probably they should not. One 
would hope, now that the annual expenditure worldwide on reticles has reached almost $\$ 3.5$ billion $^{27}$ that semiconductor manufacturers would not "turn a blind eye" to the continuing risk of reticle electrostatic damage, whether by placing undue faith in tests and instrumentation that-like Nelson's blind eye - cannot actually detect the threat, or through being reluctant to acknowledge the risk that is associated with the use of static dissipative reticle pods. The electrostatic threat to reticles is real, it is ever-present, and it is inevitable that it is continuing to cause significant losses in semiconductor production today, just as it has done for decades. The problem has not gone away, nor has it been solved by the introduction of "conductive" plastic reticle pods and boxes.

The ever-greater miniaturization of electronic devices has taken lithography from the micro to the nano regime, and the problem of reticle electrostatic damage has evolved as a result of this. Reticles used to be subject to discrete and catastrophic ESD damage events that could be easily identified, whereas they are now more likely to suffer from gradual, cumulative field-induced degradation, which is extremely difficult to diagnose as electrostatic in origin. The reduction in the rate of ESD damage in reticles since the widespread introduction of ESD countermeasures does not mean that the electrostatic threat has been adequately dealt with; the damage has just changed into something radically different in nature.

This change makes damage detection more difficult and the identification of the cause of it is now virtually impossible. Reticle damage that previously might have had an easily identifiable and rectifiable single cause, such as a broken ground connection on a wrist strap at an inspection station, now may be the result of many imperceptible low-level electrostatic stresses that are distributed throughout the reticle's handling path, some even being generated by normal operations. Reticles are inherently more susceptible to these widely distributed risk factors now, due to the continuous reduction in reticle critical dimensions over time.

Minimizing the generation of electric fields in a manufacturing environment is clearly necessary, and the static reduction measures adopted by the industry are essential, but they are not capable of fully protecting a reticle. Just as the idea of controlling the cleanliness of the entire fab environment to avoid wafer contamination was abandoned in favor of using SMIF pods and FOUPs, the approach to reticle electrostatic protection needs to move in a similar direction by making the pod adequately protective against electric fields. Doing this would actually allow static mitigation requirements in some areas to be relaxed. It would also eliminate the risks associated with human error and the failure or poor maintenance of any of the active ESD countermeasures being employed.

A reticle is a critical part of the manufacturing process that requires an extraordinary level of protection against exposure to electric field. Measurements that have only recently been possible due to the development of new sensor technology have shown that the risk to reticles from electric field is ubiquitous and is far greater than has previously been appreciated. But even these measurements have significant limitations, and they do not reveal all of the risks because they cannot adequately detect very rapidly changing fields and short duration transients over the full frequency range that can damage a reticle.
Levels of electric field that were once thought not to be problematic (as indicated by the guidance in the ITRS and SEMI Standards prior to the discovery of EFM) have subsequently been proven through calibrated experimentation to be hazardous. Recent evidence also indicates that yield losses can occur due to cumulative electrostatic degradation of the reticle even when routine reticle inspections have failed to detect any "killer defects" in the reticle.

Although the factory environment may be carefully controlled to minimize the generation of static electricity, the tribocharging of objects through normal activity is unavoidable and is capable of generating levels of electric field that can cause incremental, cumulative, and irreparable degradation in a reticle. Unfortunately, the adoption of increasingly conductive plastic materials for making reticle pods makes the risk to a reticle inside them virtually undetectable by electronic means.

So, a significant yet virtually undetectable electrostatic risk from field transients remains, and static dissipative reticle pods actually increase that risk as previously explained. The only adequate detector for the transient fields that are transmitted to a reticle by such materials is the reticle itself, but nobody in semiconductor manufacturing should want to use production reticles as test subjects, only to subsequently confirm through their demise that their reticle pod inventory is unsafe! It is more appropriate to rely on a sound scientific approach to the subject and on a thorough consideration of all the available evidence.

The evidence presented here shows conclusively that the established electrostatic protection methodology for reticles is flawed; it is neither fully effective nor is it fail-safe-it allows any failure in equipment or operational procedures to expose a reticle to electrostatic risk. Some well-established practices, which at the time when they were introduced were recommended by highly respected and experienced ESD consultants, have since been shown to actually increase the risk to reticles from electric field, rather than reducing it. Some reticle handling products from well-known manufacturers-including patented technologies that are claimed to protect reticles against electrostatic risk-are equally suspect since they are based on those same flawed principles and early misconceptions about the nature of reticle electrostatic damage.

This situation demonstrates that reticle electrostatic protection is an extremely specialized and technically difficult subject to deal with, requiring specific insights and knowledge about the interaction of electric field with reticles that many ESD consultants and engineers working in the semiconductor industry do not appear to have.

There is a proverb that says "a chain is only as strong as its weakest link," and this certainly applies to reticle protection. Getting just one link wrong in the reticle protection chain can have very serious consequences, as discovered by the semiconductor manufacturer that lost over \$1 million in 2004 through repeated damage to one particularly field-sensitive and critical production reticle. If just one example of EFM in one critical production reticle in 2004 could cost so much, the potential financial impact of such damage happening in semiconductor manufacturing today must be astronomical.

Static dissipative plastics that are widely used for making reticle pods have been shown to be a particular problem, owing to a combination of factors: 
a. Static dissipative or even "conductive" plastics do not provide adequate shielding against externally generated electric fields. By operating as a high-pass filter and selectively transmitting high frequencies to the reticle, these materials expose a reticle to a particularly hazardous aspect of electric field exposurefield transients.

b. Static dissipative plastics actually convert every change in the external field conditions around a reticle pod into an internal field transient.

c. Static dissipative plastics can be tribocharged and thus generate electric field transients that penetrate the reticle inside the pod during normal use.

(NB: Every transient creates a double stress for a reticle: once as the field increases, then again as it falls back to zero as the static charge on the plastic is dissipated).

d. "Wipe tests" that measure the retention of static charge on a pod by measuring the external field generated after rubbing with a cloth are not appropriate for judging the suitability of materials for reticle pod construction. Even the most sophisticated sensor devices that have recently been employed to study the problem of field penetration cannot detect all short-duration field transients that can be generated and transmitted by static dissipative plastics. Therefore, such tests and electronic measurements convey a false impression of the protective quality of static dissipative reticle pods.

It has been known since before the discovery of EFM that static dissipative pods do not adequately protect reticles against electric field penetration. Measurements have subsequently confirmed that static dissipative and even "conductive" plastic single-reticle pods are not sufficiently protective to prevent field-induced ESD, so even when they are used in a well-controlled factory environment with high quality ESD countermeasures in place, they are unable to prevent EFM or other field-enhanced cumulative degradation.

The only $100 \%$ effective protection for a reticle is a metallic shield, a Faraday cage. This is a well-proven, fail-safe, and passive technology. Even a partial Faraday cage, as constituted by an all-metal multi-reticle cassette, has been shown to be much more protective for reticles than the kind of static dissipative plastic single-reticle pod that has become the preferred reticle carrier in many semiconductor fabs around the world today. Since a solid metal cassette provides the electric field shielding in a multi-reticle pod, it will be equally effective across the entire frequency spectrum. This technology has been field-proven for more than 30 years.

If it is preferred that reticles are handled singly rather than in a multi-reticle pod for logistical or ergonomic reasons, the only effective and fail-safe way of providing adequate electrostatic protection is to place a metal Faraday cage around them; for example, by making the pod from metal rather than plastic. This approach has been demonstrated to be fully effective, creating a stable and protective electrostatic environment for the reticle even though the pod may be badly handled, tribocharged, or taken outside an "ESD controlled" zone.

In addition, the procedures being used when the reticle is outside the protective environment of such a pod also need to be reviewed and modified where necessary to eliminate avoidable risks that may be present there. The methodology for doing this is described in SEMI Standard E163. ${ }^{6}$

\section{Conclusions}

The standard reticle protection regime used in semiconductor manufacturing today is not effective, it is not fail-safe, and the errors and weaknesses in it will allow reticle damage to progress cumulatively.

Totally fail-safe protection against the risk of electric field-induced damage is only possible if there is a continuous metallic Faraday cage surrounding reticles. This can protect reticles against

- unavoidable electric fields arising in the handling environment,

- the consequences of charging a reticle during necessary procedures and normal use (by protecting against field perturbation until the static charge on the reticle can be safely neutralized by air ionization),

- failure or incorrect adjustment and location of active ESD countermeasures such as air ionizers,

- human error during pod handling such as forgetting to connect a garment-grounding strap, and

- taking a reticle outside an ESD-controlled environment, such as when moving a reticle between buildings or during shipment.

The static dissipative plastic single-reticle pods and boxes that are in widespread use today have been shown to expose reticles to a significant yet avoidable risk of cumulative electrostatic damage. Solutions are available to protect reticles against the risks identified in this paper, and the performance of reticle pods that are sufficiently protective to meet the latest guidance for reticle EFM protection contained in the ITRS ${ }^{26}$ (soon to be updated to the IRDS) has been demonstrated. Data showing the protective quality of an all-metal single-reticle SMIF pod have been presented. The adoption of metallic pods or even multi-reticle cassettes could reduce reliance on some of the ESD precautions that are currently adopted in semiconductor manufacturing, which require meticulous maintenance but are not fail-safe.

Semiconductor manufacturing facilities should no longer be following practices that were developed without consideration of the special field sensitivity of reticles and before the risk of EFM had been identified. Practices such as grounding reticles through static dissipative contacts, which was once believed to be protective but has subsequently been proven to increase the electrostatic risk to reticles, should not be continued. The wisdom of using static dissipative or even "conductive" plastic reticle pods is definitely called into question.

Ideally, the guidance provided in SEMI Standard E163 should be followed closely; this is the best way to minimize the risk of yield loss and to avoid the considerable cost and disruption to production that reticle electrostatic degradation due to field induction is known to cause. Newly available equipment such as the field-recording reticle used to generate much of the data presented here is invaluable in helping to identify the location of some electrostatic risk areas, but the limitations of such electronic equipment should always be 
taken into account as it cannot detect an electric field as well as the reticle itself can.

It is considered that the understanding of the behavior of electric fields described herein may also be valuable in other areas of manufacturing where static charge and field induction are problematic.

\section{Acknowledgments}

The author gratefully acknowledges Thomas Sebald of Estion GmbH, Germany, for making the E-Reticle field measurement data available and Microtome Inc., for funding and supporting the ongoing research in this field.

\section{References}

1. G. Rider, "Protection of reticles against damage from field-induced electrostatic discharge," in Semiconductor Manufacturing Magazine, 2003, http://www.sematech.org/meetings/archives/esd/20031212/index .htm.

2. G. Rider, "Reticle ESD module 1; electrostatic field simulation," in SEMATECH Reticle ESD Workshop, 2003, http://www.sematech.org/ meetings/archives/esd/20031212/SEMATECH\%20ESD1\%20short.pdf

3. A. C. Rudack et al., "Induced ESD damage on photomasks: a reticle evaluation," in EOS/ESD Symp., Las Vegas (2003).

4. D. Helmholz and M. Lering, "Reticle carrier material as ESD protection," Proc. SPIE 6349, 634952 (2006).

5. G. Rider, "Electrostatic risk to reticles in the nanolithography era," J. Micro/Nanolith. MEMS MOEMS 15(2), 023501 (2016).

6. "Guide for the handling of reticles and other extremely electrostatic sensitive (EES) items within specially designated areas," SEMI Standard E163-0212, http://ams.semi.org/ebusiness/standards/semistandard.aspx.

7. G. Rider, "EFM: a pernicious new electric field induced damage mechanism in reticles," in SEMATECH ESD Symp., 2003, http://www. sematech.org/meetings/archives/esd/20031212/index.htm.

8. G. Rider, "Quantification of the risk of field induced damage to reticles," in SEMATECH ESD Symp., 2003, http://www.sematech.org/meetings/ archives/esd/20031212/index.htm.

9. G. Rider and T. Kalkur, "Experimental quantification of reticle electrostatic damage below the threshold for ESD," Proc. SPIE 6922, 69221Y (2008).

10. G. Rider, "Electric field-induced progressive CD degradation in reticles," Proc. SPIE 7122, $71220 \mathrm{G}$ (2008).

11. A. Wallash and L. Levit, "Electrical breakdown and ESD phenomena for devices with nanometer-to-micron gaps," Proc. SPIE 4980, 87-96 (2003).
12. G. Ben-Zvi, Private Communication of data produced by Brian Grenon Consultants.

13. A. Tchikoulaeva et al., "ACLV degradation: root cause analysis and effective monitoring strategy," Proc. SPIE 7028, 702816 (2008).

14. J. Bruley et al., "Cr migration on $193 \mathrm{~nm}$ binary photomasks," Proc. SPIE 7272, 727215 (2009).

15. A. Rudack, L. Levit, and A. Williams, "Mask damage by electrostatic discharge: a reticle printability evaluation," Proc. SPIE 4691, 1340 (2002).

16. S. Labovitz et al., "Detection of progressive transmission loss due to haze with Galileo ${ }^{\mathrm{TM}}$ mask DUV transmittance mapping based on non imaging optics," Proc. SPIE 7122, 712216 (2008).

17. G. Ben-Zvi et al., "Very high sensitivity mask transmittance mapping and measurements based on non imaging optics with Galileo ${ }^{\mathrm{TM}}$," Proc. SPIE 7028, 702828 (2008).

18. P. Burlison, "Diagnosing the 'undiagnosable' faults," Future-Fab Int. 26, 113-118 (2008).

19. Private Communication, $300 \mathrm{~mm}$ Semiconductor Foundry.

20. L. Levit and G. Weil, "Reticle boxes, ESD control and electrostatic shielding," in SEMATECH ESD Impact and Control Workshop, 2001, http://www.sematech.org/meetings/archives/esd/20011020/10.pdf.

21. G. Rider, "AFM study of field induced reticle damage," in SEMATECH Reticle ESD Workshop, 2003, http://www.sematech.org/meetings/ archives/esd/20031212/SEMATECH\%20ESD2\%20short.pdf.

22. J. Montoya, L. Levit, and A. Englisch, "A study of the mechanisms for ESD damage to reticles," IEEE Trans. Electron. Package Manuf. 24(2), 78-85 (2001).

23. C. Turley, L. Kindt, and J. Kinnear Jr., "Evaluating electrostatic damage prevention methods for full-scale reticle manufacturing," in Proc. 35th Electrical Overstress/Electrostatic Discharge Symp. (2013).

24. J. Chubb, "Measuring the shielding performance of materials," in IEEE Industry Applications Conf., pp. 8-12 (2000).

25. T. Sebald and G. Rider, "High sensitivity electric field monitoring system for control of field-induced CD degradation in reticles (EFM)," Proc. SPIE 7379, 73791P (2009).

26. "International technology roadmap for semiconductors 2011 edition," https:// www.dropbox.com/sh/r51qrus06k6ehrc/AACQYSRnTdLGUCDZFhB6_ iXua/2011Chapters?dl=0\&preview=2011Factory.pdf.

27. "SEMI global update," 2017, http://www.semi.org/en/semi-reports2016-semiconductor-photomask-sales-33-billion.

Gavin C. Rider has a PhD in physics. He is currently vice president for technology and development at Microtome Inc., having previously been the product manager for material handling and factory integration with ASML in Veldhoven. He has been a key contributor in the writing of several SEMI standards and initiated the writing of SEMI Standard E163. He has coauthored several patents and has written the guidance on EFM prevention for the ITRS and IRDS. 